\title{
TU/e EmonONEN

\section{Performance study of an adaptive dual antenna handset for indoor communications}

\section{Citation for published version (APA):}

Dolmans, G., \& Leijten, L. (1999). Performance study of an adaptive dual antenna handset for indoor communications. IEE Proceedings - Microwaves, Antennas and Propagation, 146(2), 138-144.

https://doi.org/10.1049/ip-map:19990212

DOI:

10.1049/ip-map:19990212

Document status and date:

Published: 01/01/1999

\section{Document Version:}

Publisher's PDF, also known as Version of Record (includes final page, issue and volume numbers)

\section{Please check the document version of this publication:}

- A submitted manuscript is the version of the article upon submission and before peer-review. There can be important differences between the submitted version and the official published version of record. People interested in the research are advised to contact the author for the final version of the publication, or visit the $\mathrm{DOI}$ to the publisher's website.

- The final author version and the galley proof are versions of the publication after peer review.

- The final published version features the final layout of the paper including the volume, issue and page numbers.

Link to publication

\section{General rights}

Copyright and moral rights for the publications made accessible in the public portal are retained by the authors and/or other copyright owners and it is a condition of accessing publications that users recognise and abide by the legal requirements associated with these rights.

- Users may download and print one copy of any publication from the public portal for the purpose of private study or research.

- You may not further distribute the material or use it for any profit-making activity or commercial gain

- You may freely distribute the URL identifying the publication in the public portal.

If the publication is distributed under the terms of Article 25fa of the Dutch Copyright Act, indicated by the "Taverne" license above, please follow below link for the End User Agreement:

www.tue.nl/taverne

Take down policy

If you believe that this document breaches copyright please contact us at:

openaccess@tue.nl

providing details and we will investigate your claim. 


\title{
Performance study of an adaptive dual antenna handset for indoor communications
}

\author{
G.Dolmans \\ L. Leyten
}

\begin{abstract}
The focus of the paper is the design and evaluation of adaptive diversity for mobile handsets. Usually, diversity principles are optimised for base stations. However, for mobile handsets new concepts must be developed to include the size and power consumption constraints. A new modelling approach is introduced, which combines indoor radio channel simulations with circuit design parameters. This enables the inclusion of key system parameters, such as the speed of the user, the scanning speed of the antenna beams and the number of phase shifts. The radio channel simulations are based on a high-order finite-difference algorithm using the Berenger absorbing boundary condition to truncate the computational domain. The algorithm is found to be efficient and accurate enough to simulate very large structures. The analysis has resulted in an optimal design of an adaptive dual antenna handset, which combines received signals (fixed beam) while scanning the environment at the same time (scan beams). A prototype is evaluated with the numerical modelling tools and a measurement set-up. The performance is close to that of a perfect equal gain combiner.
\end{abstract}

\section{Introduction}

Previous research on (outdoor) mobile communication systems indicates that the addition of adaptive circuitry can overcome most of the system impairments caused by the effects of multipath fading [1]. Quite often, antenna diversity is implemented for this purpose at the base station. The base station is an obvious place to implement diversity, because of its size for locating multiple antennas and because of its mains power-supply which is available to drive the adaptive circuits.

For indoor communication systems the base station is located centrally in a building. Line-of-sight situations will therefore rarely occur. At the handset the angle spread of the incoming waves is $360^{\circ}$ due to the existence of many reflected waves. In this situation antenna diversity at the handset will result in an improved radio link. However, implementing antenna

\section{(C) IEE, 1999}

IEE Proceedings online no. 19990212

DOI: 10.1049/ip-map:19990212

Paper first received 5th June 1998 and in revised form 6th January 1999

The authors are with Philips Research, Prof. Holstlaan 4, WAY 51, 5656 AA Eindhoven, The Netherlands diversity in a handset is not easy due to power and size constraints. Therefore the additional circuitry should be small, efficient and not use much battery power.

In this paper a novel diversity receiver for a handset is introduced which has a performance close to that of a perfect equal-gain combiner [2]. The key features of this receiver are: the simultaneous reception of data, scanning of the propagation environment, and updating for the best signal to noise plus interference ratio $S /$ $(N+I)$. Measurement results are presented for a hybrid receiver prototype for the digital European cordless telephone (DECT) system.

To develop an adaptive antenna receiver, it is essential to predict its performance in a multipath fading environment. For this, a full-wave propagation model based on the finite-difference time-domain method (FDTD) has been developed. A new model based on the combination of Berenger's highly accurate absorbing boundary condition (ABC) together with a higherorder $(2,4)$ FDTD algorithm is proposed to model electrically large computational domains.

An important feature of this model is the possibility of analysing the diversity receiver with respect to its key performance parameters. These parameters include: the mobility of the user, the scanning speed, the number of phase shifts, the location and orientation of the antennas on the handset, etc. The combination of system and propagation modelling resulted in a diversity receiver that was optimal not only with respect to the received signal strength and interference, but also with respect to power and size constraints.

\section{Design of adaptive system}

The adaptive circuitry of the dual antenna handset is formed by a double implementation of an equal gain combiner. This circuitry is steered using a quality signal which is a measure of the signal-to-noise plus interference ratio, $S /(N+I)$.

\subsection{Quality signals}

Most adaptive antenna diversity implementations use the received signal-strength indicator (RSSI) as the quality signal to steer the adaptive circuits. This RSSI is already present in the receiver to steer the automatic gain control (AGC) circuits. The RSSI cannot discriminate between desired signals (data, speech) and interfering signals. The interfering signals can come from neighbouring telecommunication systems that operate in the same frequency band (cochannel interference) or from any other type of electronic equipment (microwave oven, computer, etc.). Changes of the RSSI level can therefore be interpreted as changes of the received signal power. Therefore, in the absence of interferers, 
the RSSI is a measure for the desired signal to noise ratio $S / N$.

The proposed system uses a different method for obtaining a quality signal $\mathrm{Q}$, which is a measure of the signal-to-noise plus interference ratio $S /(N+I)$. The method, called 'out-of-band noise detection', is based on measuring the noise power in a frequency band just above the signal band. This is implemented by a band filter, a power detector and a lowpass filter (integrator) placed behind the frequency demodulator (see Fig. 1).

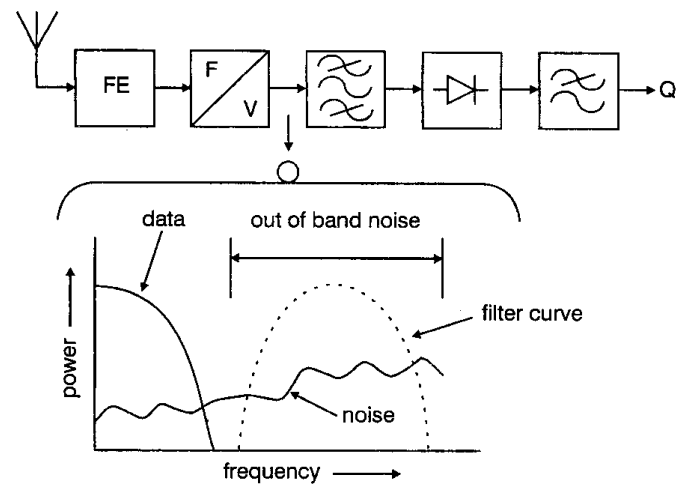

Fig. 1 Out-of-band noise detection

Top figure shows the building blocks: antenna, radio front-end (FE), frequency demodulator $(\mathrm{F} / \mathrm{V})$, band-pass filter, power detector, integrator Bottom figure shows baseband frequency signal spectrum after demodulation data $=$ baseband data frequency spectrum

noise $=$ noise frequency spectrum

filter curve $=$ filter transfer function of bandpass filter of detector

The frequency demodulator has a very large gain, but a fixed voltage swing at its output. This results in clipped (square-wave) signals or bits (zeros and ones) at the output. The input noise power has a uniform frequency distribution and has a fixed (thermal) level. At the output the noise power increases as a function of frequency, because a frequency demodulator acts as a differentiator. The data and noise frequency spectrum at the output of the demodulator are also shown in Fig. 1. Two situations, which give rise to an increased out-of-band noise power, can be distinguished: first, the input signal power decreases. Due to the very large gain of the demodulator the output signals are still clipped but the signal-to-noise ratio has decreased, resulting in an increased out-of-band noise power. Secondly, an interferer is present in the receive bandwidth. The sum of the desired and interfering signal has more zero-crossings per second than that of the desired signal alone. The frequency demodulator translates this into out-of-band high frequency components (FMclicks) which add to the out-of-band noise power. As a result of these two effects the quality signal obtained using the out-of-band noise detection is therefore a measure of the signal-to-noise plus interference ratio $S /$ $(N+I)$.

In a multipath environment with interferers, a diversity receiver with an optimal performance can be built using the proposed quality signal to steer the adaptive circuits.

\subsection{Adaptive dual antenna diversity receiver}

Fig. 2 shows a schematic diagram of the receiver. It is a selection diversity receiver based on a double implementation of the equal gain combiner. It consists of two antennas, two variable phase-shifters $\Phi 1, \Phi 2$, two summing circuits indicated by + , two receivers $\mathrm{RX1}$ and $\mathrm{RX} 2$, a microcontroller $\mu \mathrm{C}$ and a data switch [2].
The quality signals Q1 and Q2 are obtained as described in Section 2.1.

Each receiver processes the combination of the signal of one antenna and the phase shifted signal of the other. From an antenna point of view, a phase shifted combination of two antennas constitutes a phased array. The receivers are capable of forming different antenna patterns (beam scanning) due to the variable phase shifter. Some possible antenna gain patterns of two parallel dipoles a half-wavelength apart are shown in Fig. 3.

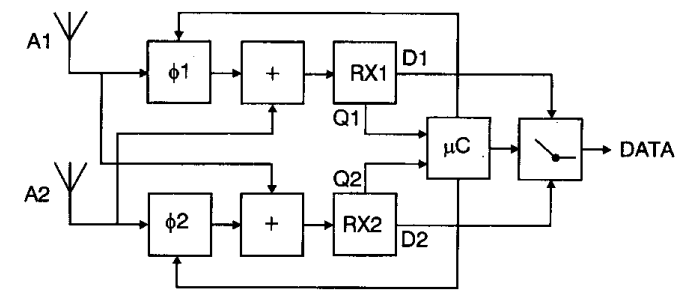

Fig.2 Schematic diagram of adaptive dual antenna receiver
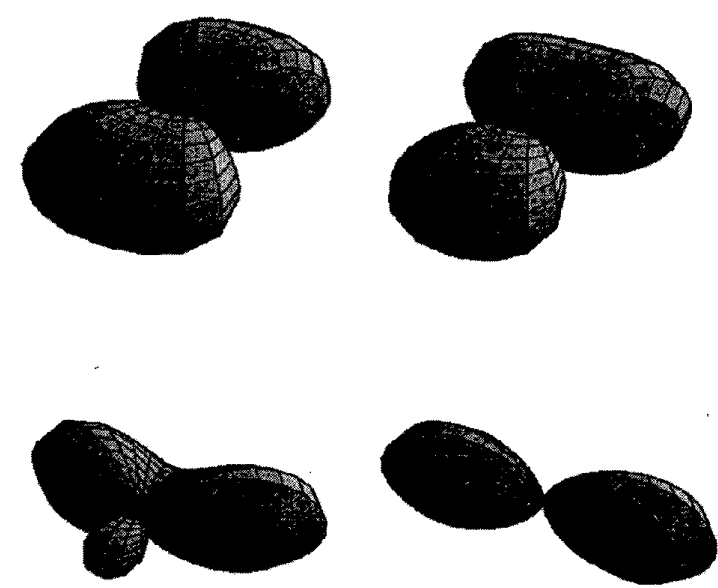

Fig.3 Antenna gain patterns of adaptive dual antenna receiver

The microcontroller steers the two phase shifters using a selection diversity algorithm and the quality signals Q1 and Q2. The algorithm starts by setting phase shifter $\Phi 1$ to a fixed level. The data D1 is switched to the output DATA. Then $\Phi 2$ runs through all its states and the quality signals Q2 of every state are compared with Q1. If a state of $\Phi 2$ is found that is better than $\Phi 1, \Phi 2$ is fixed and the data D2 is now switched to the DATA output. The scanning process repeats itself, alternately using $\Phi 1$ and $\Phi 2$ to scan for an optimal signal-to-noise plus interference ratio using the quality signals. In this way the receiver selects the best combination of the two antennas without interrupting the data stream.

The phase shifter is not implemented in the radio frequency (RF) part as shown in Fig. 2, but in the base band $(\mathrm{BB})$ part of the receiver. The integration of the phase shifter with the other BB circuits results in a small size and low cost solution. The requirements for a phase shifter in the RF part would be much higher to prevent receiver performance degradation due to increased harmonic distortion and increased noise. The feasibility of an integrated solution has been proved by a fully functional integrated circuit designed and tested within the Philips Research Laboratories. 
A discrete phase-shifter is formed by a resistor network that interpolates the $I$ and $Q$ signals and their inverted versions $\bar{I}$ and $\bar{Q}$, (see Fig. 4). Using the resistor network highly accurate signals, $\phi_{1}-\phi_{8}$, with equal amplitudes, but with $45^{\circ}$ phase steps are obtained. As shown in Fig. 2 two phase shifting circuits are necessary for the adaptive diversity receiver. The microcontroller selects one of the phase outputs of each phase shifter.

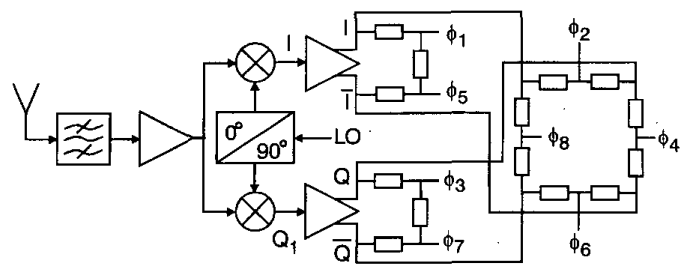

Fig.4 Eight step baseband phase shifter using resistive interpolator

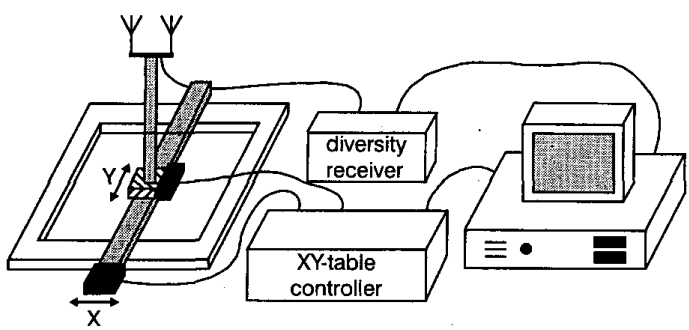

Fig.5 Measurement set-up

\section{Indoor measurements}

To measure the performance improvement of the adaptive receiver, a dedicated measurement set-up is used. It consists of a controllable XY-table that displaces the antennas in a plane of $80 \mathrm{~cm}$ by $100 \mathrm{~cm}$ (see Fig. 5). The base station is placed in a central location inside the building. The XY-table together with the diversity receiver are placed inside an arbitrarily chosen room. By moving the receive antennas on the XY-table, a realistic, varying multipath environment is created. For each point in this plane the received quality of the diversity receiver is stored on the hard-disk drive of a computer for analysis. The points in the observation plane had a separation of $1 \mathrm{~cm}$, which is less than $1 / 10$ wavelength for the $1.9 \mathrm{GHz}$ DECT frequency band.

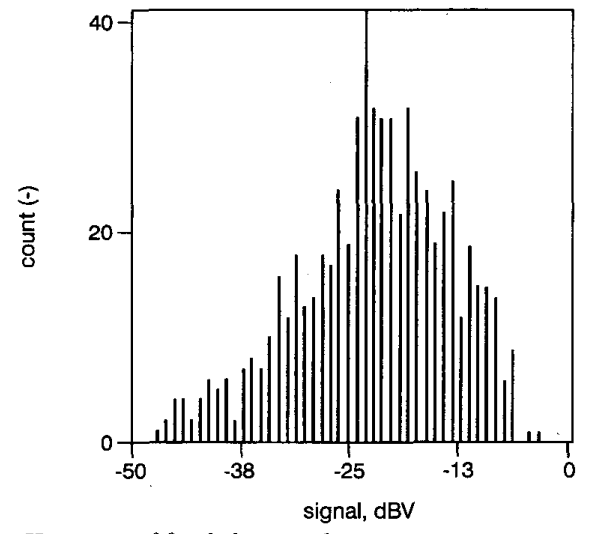

Fig. 6 Histogram of fixed phase combining

The signal levels received by the handset for a fixed phase setting and the adaptive combining technique are compared in Figs. 6 and 7. In these Figures the number of points (count) in the measurement plane are plotted as a function of the received signal strength.

From the received signal distributions it can be concluded that the mean signal level of the adaptive receiver exceeds the mean signal level of the fixed phase setting. Furthermore, the dynamic range of the received signals is significantly reduced from approximately $50 \mathrm{~dB}$ to $25 \mathrm{~dB}$

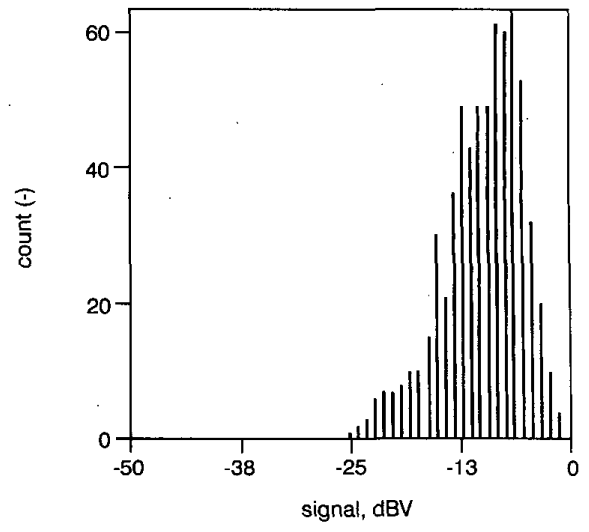

Fig. 7 Histogram of adaptive combining

\section{Propagation analysis: high-order FDTD/ Berenger}

Recently, several full-wave approaches have been proposed for solving Maxwell's equations for indoor propagation problems. The approaches are based on the modal expansion technique [3], the FDTD method [4] and the method of moments [5].

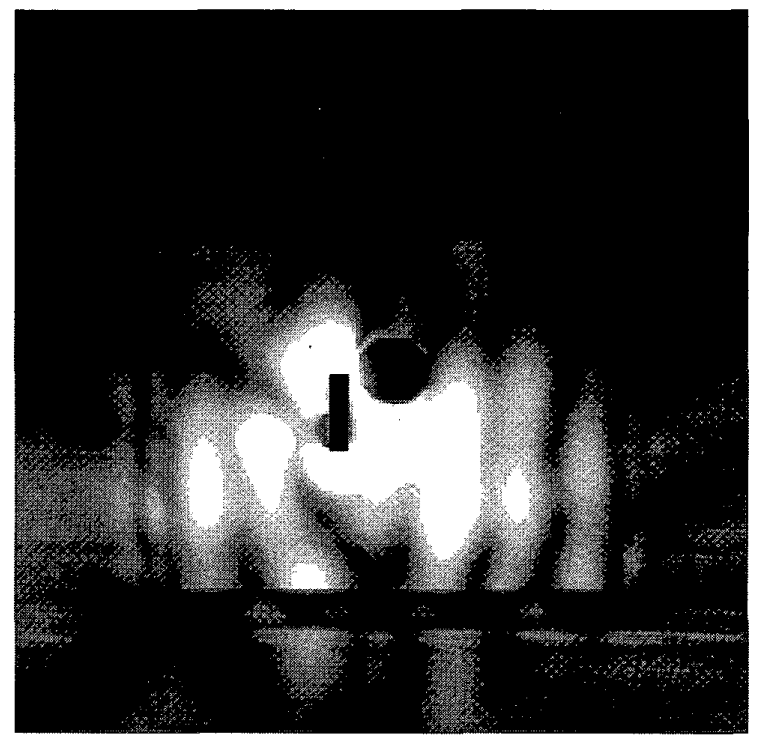

Fig.8 E-field in a small room $(2 m \times 2 m \times 2 m)$ with $(2,2)-$ Mur algorithm (3D)

\section{1 (2,2) FDTD/Mur (3D)}

The FDTD method is a well established numerical approach for the analysis of a large variety of electromagnetic problems. It is particularly useful for indoor fading radio channels because complex combinations of metallic structures and lossy dielectric material can be studied. The original FDTD algorithm which was 
second-order accurate in time and space [6], is also referred to as $(2,2)$ FDTD. The three-dimensional $(2,2)$ FDTD method truncated by second-order Mur boundary conditions [7] is capable of predicting the electromagnetic fields inside small configurations. An example of such a configuration is a small room $(2 \mathrm{~m} \times 2 \mathrm{~m} \times$ $2 \mathrm{~m}$ ) in which a handset is close to a human head.

The magnitude of the electric field is represented by a gray scale in Fig. 8. Rapid fluctuations in amplitude of the electromagnetic waves are observed for a mobile user operating in a multipath environment. This singleroom example gives a clear insight into the fluctuations, but the performance of the adaptive receiver must be analysed in an entire building. To handle these electrically large configurations, some improvements in the FDTD algorithm are necessary.

\section{2 (2,4) FDTD/Berenger (2D)}

A simulation of the electromagnetic fields in a building would lead to excessive memory requirements and computational efforts. For this case, a high-order FDTD algorithm in combination with an accurate absorbing boundary condition is chosen to reduce the memory usage and CPU time [8, 9]. Unfortunately, in these methods the absorbing boundary condition limits the overall performance of the numerical computations. In this paper, an unique combination of a $(2,4)$ FDTD algorithm and Berenger's highly accurate absorbing boundary condition [10] is employed to predict spatial and temporal electromagnetic fields in a building with rooms of various sizes.

As an example, only one component of the electric field using a fourth-order Lagrange polynomial of the spatial derivatives will be presented. The $z$-component of the electric field can be written as

$$
\begin{aligned}
& \mathcal{E}_{z}^{n}\left(k+\frac{1}{2}, l+\frac{1}{2}\right) \\
& =\frac{2 \epsilon-\sigma \Delta t}{2 \epsilon+\sigma \Delta t} \mathcal{E}_{z}^{n-1}\left(k+\frac{1}{2}, l+\frac{1}{2}\right)+\frac{2 \Delta t}{2 \epsilon+\sigma \Delta t} \\
& \frac{27}{24 \Delta x}\left\{\mathcal{H}_{y}^{n-\frac{1}{2}}\left(k+1, l+\frac{1}{2}\right)-\mathcal{H}_{y}^{n-\frac{1}{2}}\left(k, l+\frac{1}{2}\right)\right\} \\
& -\frac{1}{24 \Delta x}\left\{\mathcal{H}_{y}^{n-\frac{1}{2}}\left(k+2, l+\frac{1}{2}\right)\right. \\
& +\frac{27}{24 \Delta y}\left\{\mathcal{H}_{x}^{n-\frac{1}{2}}\left(k+\frac{1}{2}, l\right)-\mathcal{H}_{x}^{n-\frac{1}{2}}\left(k+\frac{1}{2}, l+1\right)\right\} \\
& -\frac{1}{24 \Delta y}\left\{\mathcal{H}_{x}^{n-\frac{1}{2}}\left(k+\frac{1}{2}, l-1\right)\right. \\
& \left.-\mathcal{H}_{x}^{n-\frac{1}{2}}\left(k+\frac{1}{2}, l+2\right)\right\}
\end{aligned}
$$

A numerical experiment for the (2,2)-Mur and the $(2,4)$-Berenger algorithm for a radiating source in free space close to the boundary is presented in Figs. 9 and 10.

Obviously, the radiation pattern is accurately predicted by the $(2,4)$-Berenger method. In this example, the size of each cell is $\lambda / 20$ and the frequency is $100 \mathrm{MHz}$. For a cell size of $\lambda / 3$, the $(2,4)$-Berenger method would also start to break down. The $(2,2)$-Mur method is not suitable for modelling electrically large structures, therefore, we adopted the $(2,4)$-Berenger method to predict the fading in a large building. In [11], the success of higher-order schemes to model structures that are thousands of wavelengths large is further outlined. It has been shown [4] that buildings can be treated as $2 \mathrm{D}$ propagation problems with good agreement with measured results.

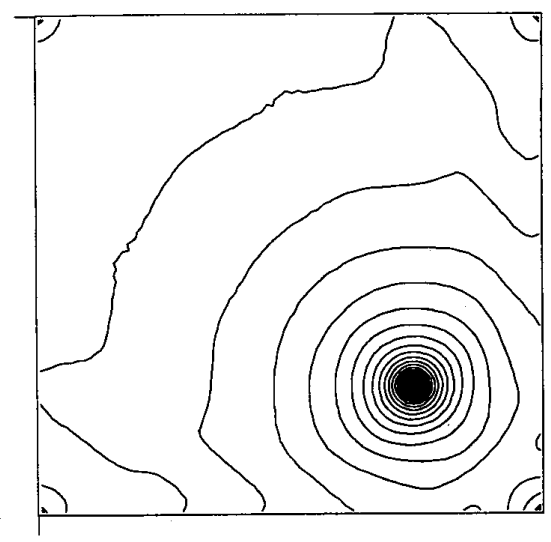

Fig.9 Radiation pattern (2D) of line-sources by (2,2)-Mur

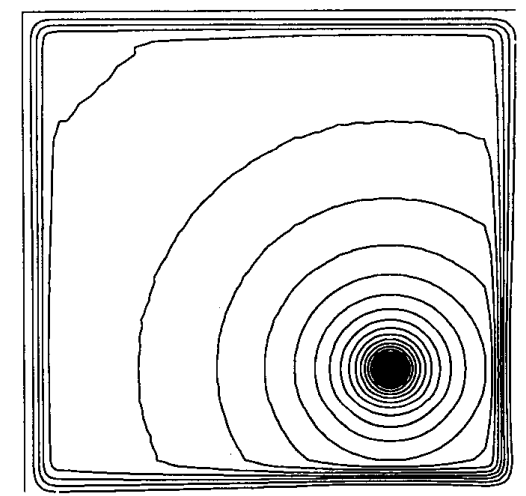

Fig.10 Radiation pattern (2D) of line-sources by (2,4)-Berenger

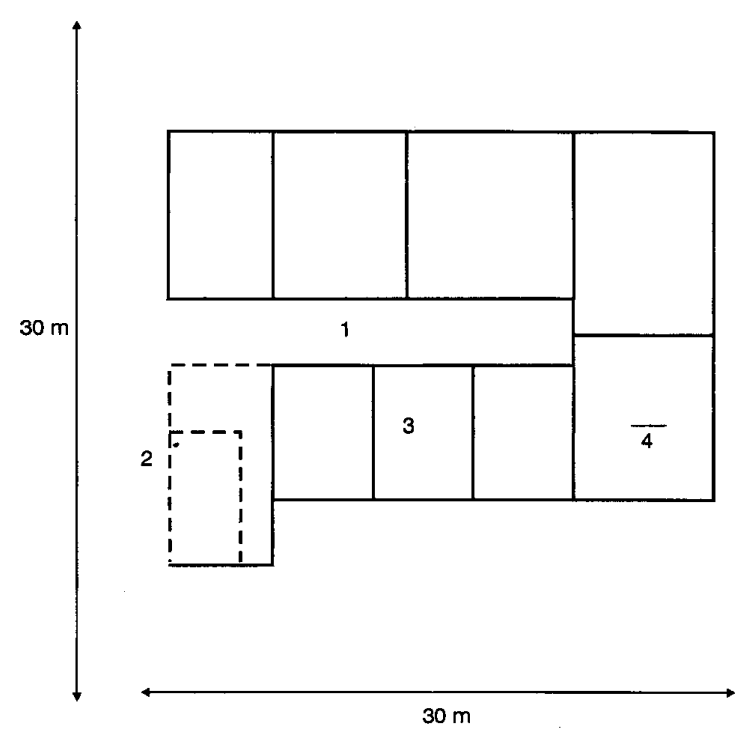

Fig. 11 Building layout of Department of Electrical Engineering, Eindhoven University of Technology

1 corridor, 2 elevator, 3 transmitter, 4 line of observation 
The two-dimensional $(2,4)$ FDTD method truncated by Berenger's boundary conditions is capable of predicting the electromagnetic fields inside a building at the Eindhoven University of Technology campus. A building blueprint is shown in Fig. 11.

The walls are modelled as isotropic, homogeneous and lossy dielectric material. The elevator on the lefthand side of the Figure is made of reflective material, therefore, this part is modelled as a shielded structure.

Fig. 12 indicates that the corridor acts as a waveguide. Furthermore, the attenuation of the walls is clearly visible in the far-field region of the configuration. At this stage in the analysis, the results of the electromagnetic field solver can be used for a circuit description of the adaptive system.

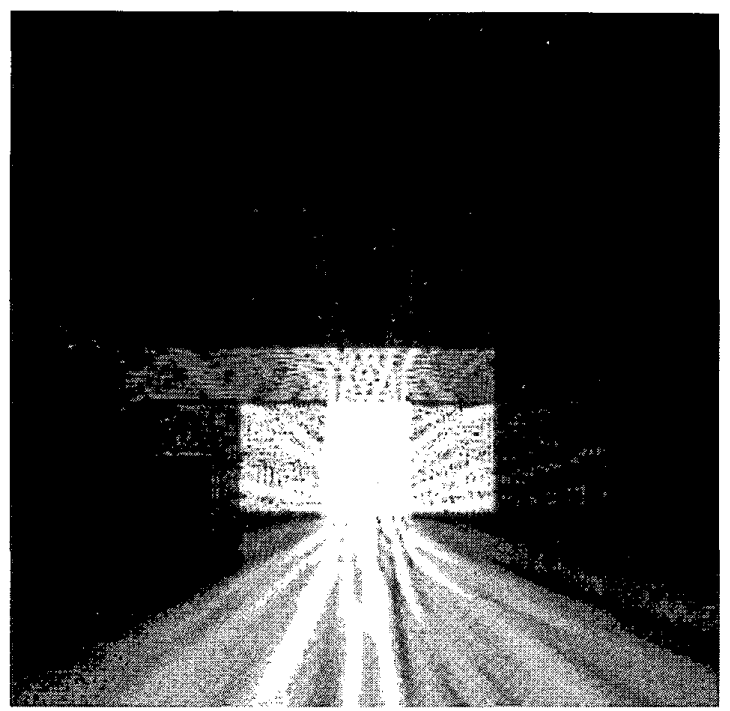

Fig.12 Amplitude fading inside and outside building at $900 \mathrm{MHz}$ Dimensions of building (2D) $30 \mathrm{~m} \times 30 \mathrm{~m}$

Location of transmitter is shown in Fig. 11

\section{System analysis using the FDTD propagation model}

Coverage predictions using the results of the FDTD simulations require information about the signal-tonoise ratios and the probability of error as a function of the movement of the mobile through the radio channel. For this purpose, the Lorentz reciprocity theorem is employed to determine the induced voltage on the used antennas [12]. A Thévenin representation with a perfectly matched load is shown in Fig. 13.

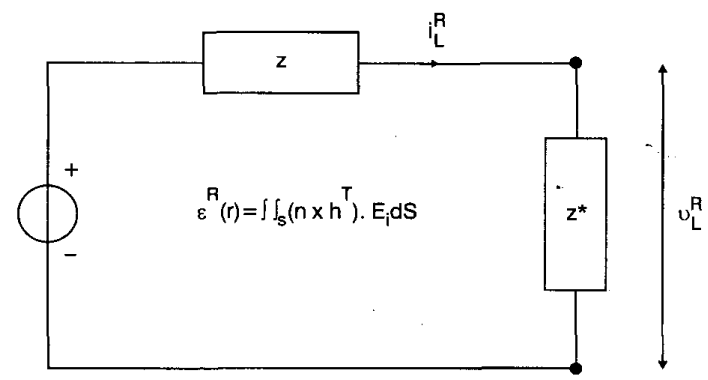

Fig.13 Thévenin representation

The voltage $\mathcal{E}^{R}(\mathbf{r})$, which is a function of the position of the mobile user in the indoor configuration, is equal to a surface integral along the wire's circumference

$$
\mathcal{E}^{R}(\mathbf{r})=\iint_{S}\left(\mathbf{n} \times \mathbf{h}^{T}\right) \cdot \mathbf{E}_{i} d S
$$

where $\mathbf{n}$ is the surface normal, $\mathbf{n} \times \mathbf{h}^{T}$ denotes the current distribution in the transmit mode of the mobile handset and $\mathbf{E}_{i}$ represents the incident electric field. Let us assume that the noise is generated by the receiver and is modelled by a zero mean stationary Gaussian random process. For a receiver using a frequency shift keying modulation standard, the relation between the signal-to-noise ratio and the probability of error $P_{e}(\mathbf{r})$ is given by

$$
P_{e}(\mathbf{r})=\frac{1}{2} \exp \frac{-S(\mathbf{r})}{2 N}=\frac{1}{2} \exp \frac{-\left|\mathcal{E}^{R}(\mathbf{r})\right|^{2}}{16 k T B \operatorname{Re}(z)}
$$

where $S$ denotes the power absorbed by the receiver and $N$ represents the noise power. The antenna impedance is given by $z$, the noise temperature is represented by $T$, Boltzmann's constant is given by $k$ and the noise bandwidth is denoted by $B$. The electric field $\mathbf{E}_{i}$ predicted by the FDTD algorithm is used to determine the induced voltage $\mathcal{E}^{R}$ on the receiving antenna. Using this system model, the coverage improvement of adaptive antennas will be studied in the following Section.

\section{Performance of adaptive dual antenna handset}

\subsection{Received signals of the adaptive dual antenna handset}

We will take a dual antenna receiver with two, four or eight phase shifts $\left(N_{\max }\right)$, a response time of $1 \mathrm{~ms}\left(t_{r}\right)$ for each phase setting and a speed for the mobile unit of $5 \mathrm{~km} / \mathrm{h}$ or $50 \mathrm{~km} / \mathrm{h}\left(c_{m}\right)$. The influence of these parameters on the performance is evaluated using the FDTD prediction.

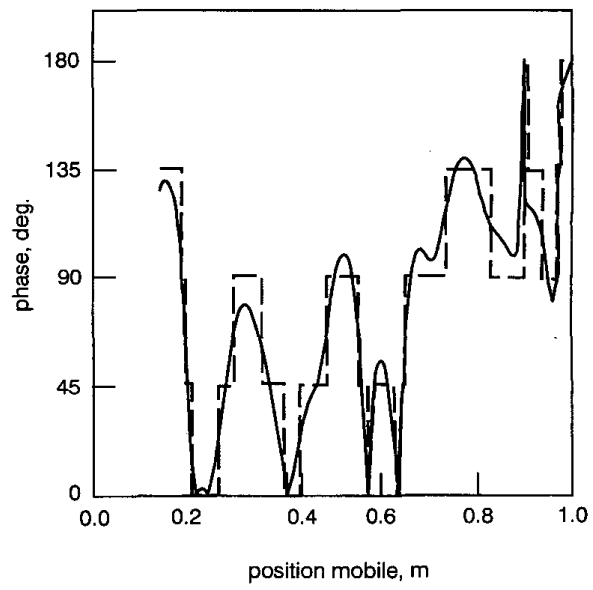

Fig. 14 Phase difference between two antennas when moving along line of observation (see Fig. 11) Field prediction is based on 2D FDTD method - actual phase difference --- corrected discrete phase difference

The adaptive handset corrects the phase difference between the two antennas so that the two signals can be combined coherently. When discrete phase levels uniformly distributed over $360^{\circ}$ are used, the phase correction is represented by $(2 \pi i) / N_{\max }$ for $i=0$.. $N_{\max }$. Fig. 14 shows the actual phase difference between two antennas inside a building. Furthermore, the corrected phase levels are shown for an adaptive handset with eight phase levels, $t_{r}$ is $1 \mathrm{~ms}$ and the speed of the user $c_{m}$ 
is $5 \mathrm{~km} / \mathrm{h}$. A nonoptimal phase influences the diversity gain and the received signal strength. The received signal strength is shown in Fig. 15.

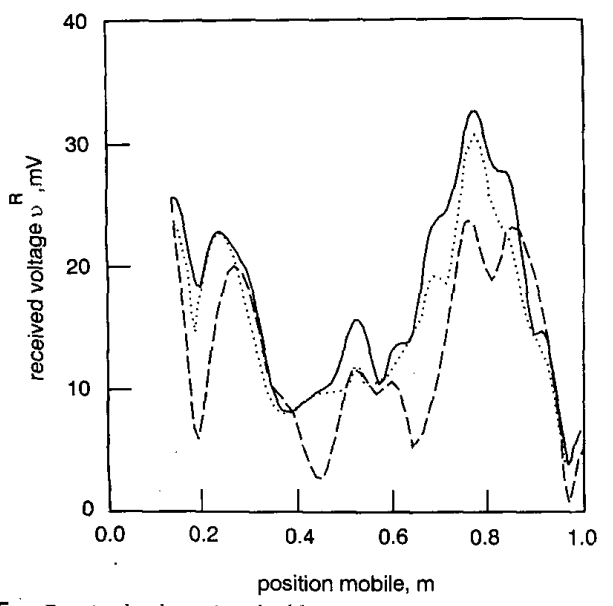

Fig. 15 Received voltage in a building theoretical diversity combiner

- - - single antenna system

.... adaptive dual antenna system when $N_{\max }=2, t_{r}=1 \mathrm{~ms}, c_{m}=5 \mathrm{~km} / \mathrm{h}$

An almost optimal signal strength is achieved with only two phase shifts. It is most important that the minimum values in the received signal strengths are almost equal for the theoretical diversity combiner and the implemented adaptive dual antenna system. This indicates that an optimal diversity gain should be possible with the proposed algorithm.

\subsection{Coverage of the adaptive dual antenna handset for several speeds}

The diversity gain, which is a measure of the reduction of the spatial 'dead spots', will be evaluated for the adaptive dual antenna receiver. The following procedure for the performance evaluation of the adaptive receiver is used:

(1) In the building, presented in Fig. 11, an area will be defined which should be covered by the transmitter. The electric field $\mathbf{E}_{i}(\mathbf{r})$ and the induced voltage $v^{R}$ on the dual antenna system is predicted with the FDTD method in this area. In practice, a distance of $1 \mathrm{~cm}$ between the grid points in the area is sufficient to reveal the rapid fluctuations.

(2) The received signal-to-noise ratio $S / N$ and probability of error $P_{c}$ are calculated at each point in the coverage area. Then, the coverage will be defined as a percentage of the total area for which the average probability of error is better than a predefined threshold. This means that the coverage is given by

$$
\text { coverage: } \#\left(P_{e}(\mathbf{r})<P_{e A V}\right) / \# \text { total area }
$$

(3) The diversity gain will be defined as the difference between the power transmitted for a nondiversity system $\left(P_{t n}\right)$ and the power transmitted in the adaptive diversity case $\left(P_{t d}\right)$ to yield a coverage of $99 \%$. This can be written as

$$
\text { diversity gain: } P_{t n}(99 \%)-P_{t d}(99 \%)
$$

The coverage against the transmitted power of the base station is calculated for an adaptive mobile handset moving at speeds of $5 \mathrm{~km} / \mathrm{h}$ and $50 \mathrm{~km} / \mathrm{h}$ in Fig. 16 .

At a speed of $5 \mathrm{~km} / \mathrm{h}$, the performance is almost equal to the perfect equal gain combiner, which results in a diversity gain of approximately $9 \mathrm{~dB}$ at a coverage of $99 \%$. For a mobile user moving at a high speed of $50 \mathrm{~km} / \mathrm{h}$, differences between the adaptive receiver and the theoretical diversity combiners can be observed. To our surprise, only two phase shifts are sufficient to realise an almost optimal diversity gain.

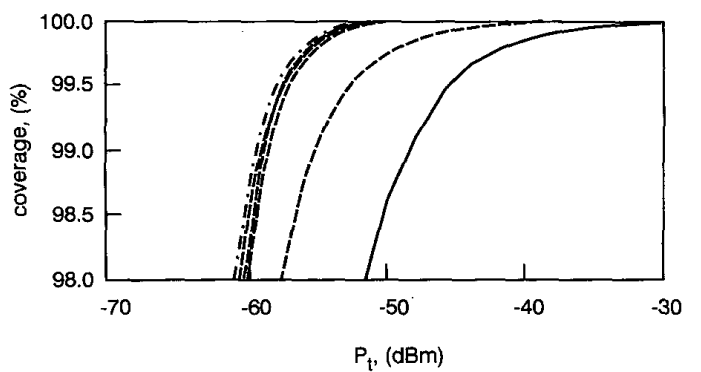

Fig. 16 Coverage plotted against power for a mobile speed - single antenna receiver --- adaptive receiver moving at $50 \mathrm{~km} / \mathrm{h}$ Other curves: adaptive receiver with 2,4 or 8 phase shifts at $5 \mathrm{~km} / \mathrm{h}$ with a theoretical equal gain receiver

\section{Conclusions}

It can be concluded that implementing diversity in a handset is feasible and the obtained improvement in system performance is significant.

A new diversity handset has been designed using a new modelling approach that includes electromagnetic simulation with circuit design. This approach provides a general framework in which adaptive antenna systems can be evaluated in both narrowband and wideband signal environments. The approach includes key system parameters such as: speed of the user, scanning speed of the antenna beams and the number of phase shifts.

The newly developed diversity handset combines the received signals to form a fixed beam and simultaneously creates a scanning beam. It uses 'out-of-band noise detection' to ensure that antenna patterns are changed when experiencing different signal-to-noise and interference conditions. It has a diversity gain of $9 \mathrm{~dB}$ at a coverage of $99 \%$ for normal walking speeds, i.e. around $5 \mathrm{~km} / \mathrm{h}$.

\section{Acknowledgments}

The authors wish to thank their colleagues at the Philips Research Laboratories, Eindhoven, for their support.

\section{References}

1 FUJIMOTO, K.: 'Overview of antenna systems for mobile communications and prospects for the future technology', IEICE Trans., 1991, E. 74, (10), pp. 3191-3201

2 TOMBEUR A WAGEMANS A BALTUS, P LEYTEN, L. and van SINDEREN, J.: 'A radio transmission system and a radio apparatus for use in such a system'. European patent application EP 0728372, 1996

3 KIM, K.-S., WHITMAN, G.M., and NIVER, E.: 'A theoretical model for radio signal attenuation inside buildings', IEEE Trans., 1995, VT-44, (3), pp. 621-629

4 BAHR, A., LAUER, A., and WOLFF, I.: 'Fdtd simulations of indoor propagation'. Proceedings of the 44th IEEE Vehicular
technology conference, Stockholm, Sweden, 1994, pp. 883-886

5 OLYSLAGER, F., DE BACKER, B., and DE ZUTTER, D.: 'An integral equation approach to the prediction of wave propagation in an indoor environment'. IEEE 3rd symposium on Communications and Vehicle Technology in the Benelux, Eindhoven, The Netherlands, 1995 , pp. 28-33

$6 \mathrm{YEE}$ K S . 'Numerical solution of initial boundary value problems involving Maxwell's equations in isotropic media', IEEE Trans., 1966, AT-14, (3), pp. 302-307 
7 MUR, G.: 'Absorbing boundary conditions for the finite-difference approximation of the time-domain electromagnetic-field equations', IEEE Trans., 1981, EC-23, (4), pp. 377-382

8 PETROPOULOS, P.G.: 'Phase error control for fdtd methods of second and fourth order accuracy', IEEE Trans., 1994, AP-42, (6), pp. 859-862

9 BROSCHAT, S.L., MANRY, C.W., and SCHNEIDER, J.B. 'Higher-order fdtd methods for large problems', Appl. Comp. Elec. Soc. J., 1995, 10, (2), pp. 17-29
10 BERENGER, J.P.: 'A perfectly matched layer for the absorption of electromagnetic waves', J. Comput. Phys., 1994, 114, pp. 185200

11 HADI, M.F., and PIKET-MAY, M.: 'A modified fdtd $(2,4)$, scheme for modeling electrically large structures with high-phase accuracy', IEEE Trans., 1997, AP-45, pp. 254-264

12 DOLMANS, W.M.C.: 'Effect of indoor fading on the performance of an adaptive antenna system'. $\mathrm{PhD}$ thesis, Eindhoven University of Technology, 1997 